\title{
Histopathological criteria for additional treatment after endoscopic mucosal resection for esophageal cancer: analysis of 464 surgically resected cases
}

Takako Eguchi ${ }^{1,2,3}$, Yukihiro Nakanishi ${ }^{4}$, Tadakazu Shimoda ${ }^{1}$, Motoki Iwasaki ${ }^{5}$, Hiroyasu Igaki ${ }^{6}$, Yuji Tachimori ${ }^{6}$, Hoichi Kato ${ }^{6}$, Hajime Yamaguchi ${ }^{2}$, Daizo Saito ${ }^{2}$ and Satoshi Umemura ${ }^{3}$

${ }^{1}$ Clinical Laboratory Division, National Cancer Center Hospital, Tokyo, Japan; ${ }^{2}$ Endoscopy Division, National Cancer Center Hospital, Tokyo, Japan; ${ }^{3}$ Second Department of Internal Medicine, Yokohama City University, Kanagawa, Japan; ${ }^{4}$ Pathology Division, National Cancer Center Research Institute, Tokyo, Japan; ${ }^{5}$ Epidemiology and Prevention Division, National Cancer Center, Research Center for Cancer Prevention and Screening, Tokyo, Japan and ${ }^{6}$ Surgery Division, National Cancer Center Hospital, Tokyo, Japan

\begin{abstract}
No previous reports on lymph-node metastasis (LNM) from superficial squamous cell carcinoma of the esophagus have proposed definite criteria for additional treatment after endoscopic mucosal resection (EMR). We investigated the association between histopathological factors and LNM in 464 consecutive patients with superficial squamous cell carcinoma of the esophagus who had undergone a radical esophagectomy with lymph-node dissection (14 'M1' lesions: intraepithelial tumors, 36 'M2' lesions: tumors invading the lamina propria, 50 'M3' lesions: tumors in contact with or invading the muscularis mucosa, 32 'SM1' lesions: tumors invading the most superficial $1 / 3$ of the submucosa and 332 'SM2/3' lesions: tumors invading deeper than SM1 level). Histopathological factors including invasion depth, size, lymphatic invasion (LY), venous invasion, tumor differentiation, growth pattern, degree of nuclear atypia and histological grade were assessed for their association with LNM in 82 M3 or SM1 lesions to determine which patients need additional treatment after EMR. LNM was found in $0.0,5.6,18.0,53.1$ and $53.9 \%$ of the M1, M2, M3, SM1 and SM2/3 lesions, respectively. A univariate analysis showed that each of the following histopathological factors had a significant influence on LNM: invasion depth (M3 vs SM1), LY, venous invasion and histological grade. Invasion depth and LY were significantly associated with LNM in a multivariate analysis. Four out of 38 patients $(10.3 \%)$ with M3 lesions without LY had LNM, whereas five out of 12 patients $(41.7 \%)$ with M3 lesions and LY had LNM. Only patients with M1/2 lesions are good candidates for EMR. Invading the muscularis mucosa (M3) is a high-risk condition for LNM the same as submucosal invasion, but M3 lesions without LY can be followed up after EMR without any additional treatment.
\end{abstract}

Modern Pathology (2006) 19, 475-480. doi:10.1038/modpathol.3800557; published online 27 January 2006

Keywords: histopathological study; endoscopic mucosal resection; lymph-node metastasis; squamous cell carcinoma; superficial esophageal carcinoma

The proportion of patients with superficial squamous cell carcinoma of the esophagus (SSCCE) and lymph-node metastasis (LNM) has been reported to be $26-53 \%$, whereas the proportion of SSCCE patients with intraepithelial carcinoma or carci-

Correspondence: Dr Y Nakanishi, MD, PhD, Pathology Division, National Cancer Center Research Institute, 5-1-1 Tsukiji, Chuo-ku, Tokyo 104-0045, Japan.

E-mail: yknakani@gan2.ncc.go.jp

Received 7 October 2005; revised and accepted 27 December 2005; published online 27 January 2006 noma invading the lamina propiria and LNM is only $0-5 \% .{ }^{1-6}$ Consequently, intraepithelial carcinoma or carcinoma invading the lamina propria is generally treated by endoscopic mucosal resection (EMR). ${ }^{1-6}$ Radical esophagectomy with lymph-node dissection is still regarded as an invasive treatment, given the high operative mortality and morbidity rates. ${ }^{6-11}$ In addition, the poor quality of life of patients after such aggressive surgery prompted us to search for expanded indications for EMR. No previous reports, however, have proposed definite criteria for additional treatment after EMR in patients with 
SSCCE because of the small number of cases in these studies, inconsistent methods of lymph-node dissection, inconsistent evaluations of invasion depth or inconsistent histological typing, including not only squamous cell carcinoma but also adenocarcinoma. ${ }^{1-4,6}$

We previously evaluated a total of 240 patients with SSCCE, including 20 patients with 'M3' lesions (ie tumors in contact with or invading the muscularis mucosa) and 166 patients with 'SM' lesions (ie tumors invading the submucosa) to clarify the association between histopathological findings and LNM. ${ }^{3}$ Lymphatic invasion (LY) was significantly associated with LNM in a multivariate analysis, and LY and histological grade (HG) were found to be prognostic factors in patients with SSCCE. Furthermore, none of the 21 lesions with HG1/2 and a vertical tumor invasion depth from the muscularis mucosa (VTID) of $<200 \mu \mathrm{m}$ without LY or venous invasion (V) had LNM. Therefore, our previous study concluded that those 21 lesions could be treated by EMR.

In this study, we reanalyzed the histopathological features of patients with SSCCE to investigate whether histopathological criteria for additional treatment after EMR could be determined based on associations between histopathological findings and LNM in a larger population of patients with SSCCE. We especially focused on minimally invasive lesions such as M3 and SM1 lesions, which can be resected by EMR without leaving any cancer tissue in the esophagus.

\section{Materials and methods}

\section{Patients}

Our series consisted of 464 consecutive patients who had undergone a radical esophagectomy with lymphadenectomy, either thoraco-abdominal (twofield) or cervico-thoraco-abdominal (three-field), for the treatment of SSCCE at the National Cancer Center Hospital in Tokyo, Japan, between January 1980 and October 2004. The histopathological features of 255 of these patients were reported in our previous report. ${ }^{3}$ No preoperative radiotherapy, chemotherapy or EMR was performed in the present series. The patients comprised 415 (89.4\%) men and $49(10.6 \%)$ women. The mean age was 61.7 years (range: 40-86 years). We classified the SSCCE lesions based on the invasion depth as follows: 'M1' for intraepithelial carcinomas; 'M2' for tumors invading the lamina propria; 'M3' for tumors in contact with or invading the muscularis mucosa; 'SM1' for tumors invading the most superficial $1 / 3$ of the submucosa; and 'SM2/3' for tumors invading deeper than the SM1 level (Figure 1). The association between LNM and clinical outcome was examined in each group. In addition, the histopathological findings described below were analyzed using $82 \mathrm{M} 3$ or SM1 lesions to determine

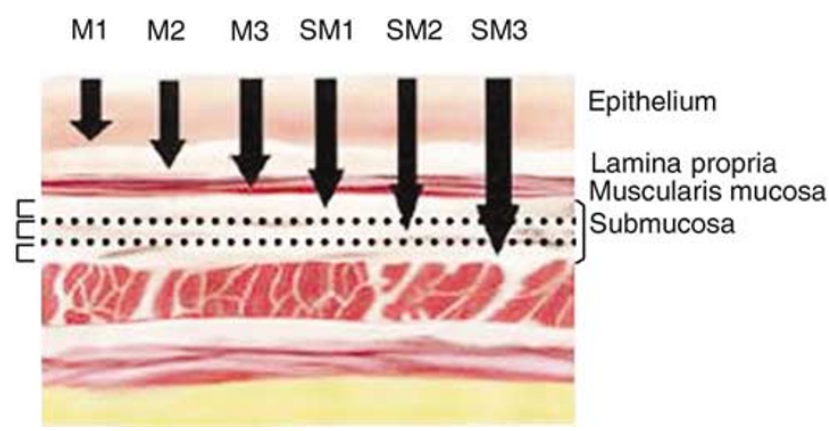

Figure 1 Subdivision of superficial esophageal carcinoma according to depth of invasion. M1: intraepithelial tumors; M2: tumors invading the lamina propria; M3: tumors in contact with or invading the muscularis mucosa; SM1: tumors invading the most superficial 1/3 of the submucosa; SM2/3: tumors invading deeper than the SM1 level.

which patients required additional treatment after EMR. When a patient had multicentric esophageal carcinomas, the histopathological factors for the lesion with the greatest invasion depth were evaluated.

\section{Histopathological Review}

Serial 5-mm-thick tissue sections of the entire lesion were cut from resected specimens fixed with $10 \%$ buffered formalin, embedded in paraffin and stained with hematoxylin and eosin using standard methods. The invasion depth- VTID, tumor size, LY, V, LNM, tumor differentiation, growth pattern, degree of nuclear atypia and $\mathrm{HG}^{3}$ were histopathologically evaluated. The distance from the invasive front to the muscularis mucosa was measured using microscopy with an ocular lens scale and defined as VTID. In specimens where the muscularis mucosa was disrupted by ulceration or tumor invasion, the muscularis mucosa level was estimated by drawing a line to connect the remaining muscularis mucosa. Tumor differentiation was assessed according to the World Health Organization classification. ${ }^{12}$ The degree of nuclear atypia was classified as low (sharp nuclei of uniform size with homogeneous chromatin: one point) or high (polymorphic nuclei with vesicular or dot-like chromatin: two points). The growth pattern was classified as expansive (large solid nests with a clear tumor margin: one point) or infiltrative (small nests and dissociated cells with an unclear tumor margin: two points). The degree of nuclear atypia and growth pattern were evaluated at the deepest area of the invading tumor. The HG of each tumor was calculated in the same manner as in our previous report by adding the nuclear atypia and growth pattern scores to obtain Grade 1: two points; Grade 2: three points; or Grade 3: four points. ${ }^{3}$ Independent histopathological reviews were performed by two experienced pathologists (YN and TS) who had no prior knowledge of the clinical 
outcomes of the patients. Their results were compared, and any discrepancies were resolved by consensus at a meeting following a further histopathological review.

\section{Statistical Analysis}

An unconditional logistic regression analysis was performed using the SAS LOGISTIC procedure (SAS Institute Inc., Cary, NC, USA) to examine the association between the histopathological factors and LNM. All $P$-values were two-sided, and the significance level was set at $P<0.05$.

\section{Results}

\section{Clinicopathological Features Based on Invasion Depth}

LNM was found in none of 14 patients with M1 lesions, two of the 36 patients (5.6\%) with M2 lesions, nine of the 50 patients $(18.0 \%)$ with M3 lesions, 17 of the 32 patients $(53.1 \%)$ with SM1 lesions and 179 of the 332 patients (53.9\%) with SM2/3 lesions. The number of dissected lymph nodes in each case was $48 \pm 21$ (mean \pm standard deviation). None of the patients with M1, M2, M3 or SM1 lesions died of esophageal carcinoma, whereas 63 patients $(40.7 \%)$ with SM2/3 lesions died from their primary disease. Six of the 464 patients (0.2\%) died within 1 month of surgery owing to surgical complications. The median follow-up duration was 4.2 years (range: 2 days-23.5 years).

\section{Clinical Characteristics of 82 Patients with M3 and SM1 Lesions}

The clinical characteristics of 82 patients with M3 and SM1 lesions are shown in Table 1. Multicentric synchronous esophageal carcinomas were found in 30 patients $(36.6 \%)$, whereas 28 patients $(34.2 \%)$ had synchronous or metachronous multiple malignancies in other organs. The VTID was $130 \pm 106 \mu \mathrm{m}$ (mean \pm standard deviation) for the SM1 lesions.

\section{Relationships between Histopathological Findings and Lymph-Node Metastasis in Patients with M3 and SM1 Lesions}

The relationships between the histopathological findings and LNM in 82 patients with M3 and SM1 lesions were determined using a univariate analysis and are shown in Table 2. The univariate analysis showed that each of the following histopathological factors had a significant influence on LNM: invasion depth (M3 vs SM1), LY, V and HG (odds ratios $(\mathrm{OR})=5.16,4.60,10.0$ and 3.76, respectively). A multivariate analysis revealed that only invasion depth and LY were independently correlated with LNM $(\mathrm{OR}=4.71$ and 3.83 , respec-
Table 1 Clinical characteristics of 82 patients with M3 or SM1 lesions

\begin{tabular}{lcr}
\hline Characteristics & Number & $\%$ \\
\hline Location & & \\
Cervical & 2 & 2.4 \\
Upper thoracic & 14 & 17.1 \\
Middle thoracic & 50 & 61.0 \\
Lower thoracic & 15 & 18.3 \\
Abdominal & 1 & 1.2 \\
& & \\
Circumference & & \\
$<25 \%$ & 26 & 31.7 \\
$\geq 25 \%<50 \%$ & 21 & 25.6 \\
$\geq 50 \%<75 \%$ & 20 & 24.4 \\
$\geq 75 \%<100 \%$ & 10 & 12.2 \\
100\% & 5 & 6.1 \\
& & \\
Number of synchronous esophageal carcinomas & \\
Solitary & 52 & 63.4 \\
Multiple & 30 & 36.6 \\
& & \\
Synchronous or metachronous malignancies of other organs \\
Absent & 54 & 65.9 \\
Present & 28 & 34.2 \\
$\quad$ Head and neck & 17 & 45.9 \\
Stomach & 10 & 27.0 \\
Lung & 3 & 8.1 \\
Colon and rectum & 2 & 5.4 \\
Prostate & 2 & 5.4 \\
Breast & 1 & 2.7 \\
Kidney & 1 & 2.7 \\
Lymphoma & 1 & 2.7 \\
malignancies in 28 patients & & \\
\hline
\end{tabular}

M3: carcinoma in contact with or invading the muscularis mucosa; SM1: carcinoma invading the most superficial $1 / 3$ of the submucosa.

tively; $95 \%$ confidence interval $=1.55-14.3$ and 1.18-12.4, respectively) (Table 3). Four patients out of a total of 38 patients $(10.3 \%)$ with M3 lesions but without LY had LNM, whereas five out of 12 patients $(41.7 \%)$ with M3 lesions and LY had LNM. Six patients out of a total of 21 patients $(28.6 \%)$ with SM1 lesions but without LY had LNM, whereas all the 11 patients (100\%) with SM1 lesions and LY had LNM (Table 4).

\section{Discussion}

LNM was observed in $0.0 \%$ of patients with M1 lesions, $5.6 \%$ of patients with M2 lesions, $18.0 \%$ of patients with M3 lesions, 53.1\% of patients with SM1 lesions and $53.9 \%$ of patients with SM2/3 lesions. As $96 \%$ of patients with M1/2 lesions developed no LNM, EMR alone can be a definitive treatment for patients with M1 and M2 lesions, taking into account the operative mortality, morbidity and poor quality of life after surgery. ${ }^{6-11}$ In contrast, more than half of the patients with SM1 or SM2/3 lesions developed LNM; therefore, these patients should receive additional systemic treatments after EMR. The proportion of patients with M3 lesions who developed LNM was not negligible; 
Table 2 Proportion of patients and crude OR for lymph-node metastasis according to histopathological findings in patients with M3 or SM1 lesions (82 cases)

\begin{tabular}{|c|c|c|c|c|c|c|}
\hline & \multicolumn{2}{|c|}{ Positive LNM $(\mathrm{n}=26)$} & \multicolumn{2}{|c|}{ Negative LNM $(\mathrm{n}=56)$} & \multirow[t]{2}{*}{ OR } & \multirow[t]{2}{*}{$95 \% C I$} \\
\hline & Number & $\%$ & Number & $\%$ & & \\
\hline \multicolumn{7}{|l|}{ Invasion depth } \\
\hline M3 & 9 & 18.0 & 41 & 82.0 & 1.00 & \\
\hline SM1 & 17 & 53.1 & 15 & 46.9 & 5.16 & $1.90,14.1$ \\
\hline \multicolumn{7}{|l|}{ Tumor size (mm) } \\
\hline$<25$ & 6 & 24.0 & 19 & 76.0 & 1.00 & \\
\hline$\geq 25$ and $<45$ & 11 & 45.8 & 13 & 54.2 & 2.68 & $0.79,9.07$ \\
\hline$\geq 45$ & 9 & 27.3 & 24 & 72.7 & 1.19 & $0.36,3.92$ \\
\hline \multicolumn{7}{|c|}{ Lymphatic invasion (LY) } \\
\hline Negative & 13 & 22.0 & 46 & 78.0 & 1.00 & \\
\hline Positive & 13 & 56.5 & 10 & 43.5 & 4.60 & $1.64,12.9$ \\
\hline \multicolumn{7}{|l|}{ Venous invasion (V) } \\
\hline Negative & 22 & 28.6 & 55 & 71.4 & 1.00 & \\
\hline Positive & 4 & 80.0 & 1 & 20.0 & 10.0 & $1.06,94.5$ \\
\hline \multicolumn{7}{|l|}{ Tumor differentiation } \\
\hline Well to moderately & 21 & 29.2 & 51 & 70.8 & 1.00 & \\
\hline Poorly & 5 & 50.0 & 5 & 50.0 & 2.43 & $0.64,9.27$ \\
\hline \multicolumn{7}{|l|}{ Growth pattern } \\
\hline Expansive & 22 & 30.1 & 51 & 69.9 & 1.00 & \\
\hline Infiltrative & 4 & 44.4 & 5 & 55.6 & 1.86 & $0.45,7.57$ \\
\hline \multicolumn{7}{|l|}{ Degree of nuclear atypia } \\
\hline Low & 21 & 27.3 & 56 & 72.7 & 1.00 & \\
\hline High & 5 & 100.0 & 0 & 0.0 & - & - \\
\hline \multicolumn{7}{|l|}{ Histological grade (HG) } \\
\hline Grade 1 & 19 & 27.1 & 51 & 72.9 & 1.00 & \\
\hline Grade $2 / 3$ & 7 & 58.3 & 5 & 41.7 & 3.76 & $1.06,13.3$ \\
\hline
\end{tabular}

LNM: lymph-node metastasis; OR: odds ratio; 95\% CI: 95\% confidence interval; M3: carcinoma in contact with or invading the muscularis mucosa; SM1: carcinoma invading the most superficial $1 / 3$ of the submucosa.

Table 3 Multivariate OR for lymph-node metastasis according to histopathological findings in patients with M3 or SM1 lesions (82 cases)

\begin{tabular}{|c|c|c|}
\hline & $O R^{\mathrm{a}}$ & $95 \% C I$ \\
\hline \multicolumn{3}{|c|}{ Invasion depth } \\
\hline M3 & 1.00 & \\
\hline SM1 & 4.71 & $1.55,14.3$ \\
\hline \multicolumn{3}{|c|}{ Lymphatic invasion (LY) } \\
\hline Negative & 1.00 & \\
\hline Positive & 3.83 & $1.18,12.4$ \\
\hline \multicolumn{3}{|c|}{ Venous invasion (V) } \\
\hline Negative & 1.00 & \\
\hline Positive & 3.02 & $0.28,33.1$ \\
\hline \multicolumn{3}{|c|}{ Histological grade (HG) } \\
\hline Grade 1 & 1.00 & \\
\hline Grade $2 / 3$ & 3.19 & $0.78,13.0$ \\
\hline
\end{tabular}

Table 4 Proportion of patients for lymph-node metastasis according to the invasion depth and lymphatic invasion in patients with M3 or SM1 lesions (82 cases)

\begin{tabular}{lccc}
\hline & M3 & SM1 & Total \\
\hline $\mathrm{LY}(-)$ & $4 / 38(10.3 \%)$ & $6 / 21(28.6 \%)$ & $10 / 59(16.9 \%)$ \\
$\mathrm{LY}(+)$ & $5 / 12(41.7 \%)$ & $11 / 11(100 \%)$ & $16 / 23(69.6 \%)$ \\
\hline
\end{tabular}

M3: carcinoma in contact with or invading the muscularis mucosa; SM1: carcinoma invading the most superficial $1 / 3$ of the submucosa; LY: lymphatic invasion.

therefore, we tried to determine any associations in the histopathological features of M3 lesions and LNM. Invasion depth (M3 vs SM1) and LY were significantly associated with LNM in a multivariate analysis $(\mathrm{OR}=4.71$ and 3.83 , respectively; 95\% confidence interval $=1.55-14.3$ and $1.18-12.4$, respectively). Because LY might be a predictive factor for LNM, LY should be precisely examined in resected specimens. Almost half of the patients 
with M3 lesions and LY had LNM; therefore, these lesions should be treated as a systemic disease, like SM lesions. Regarding the M3 lesions without LY, LNM was found in $10.3 \%$ of the patients (four out of 38 cases). Patients with M3 lesions without LY should decide whether they won't undergo additional treatment after EMR or they will undergo additional systemic treatments such as esophagectomy or chemo-radiation therapy after EMR, considering the possibility of LNM and the invasiveness of systemic therapy, after receiving a thorough explanation of the possibility of LNM and the invasiveness of systemic therapy and providing their informed consent. Although we tried to find patients who did not require additional systemic treatment after EMR for SM1 lesions in order to expand the current criteria for EMR, rather limited criteria were established based on the results of the present study. In this study, we examined a large number of patients with SSCCE who had been treated using the same approach (esophagectomy with two- or three-field lymphnode dissection) and whose resected specimens had been examined in the same manner. Thus, the present study provides reliable data concerning LNM in patients with SSCCE treated at a single institution.

In the present analysis, the proportions of patients with LNM in each group were higher than those reported in previous reports. ${ }^{1-6}$ This discrepancy might be explained by the fact that all the patients in the present study underwent a radical esophagectomy with two- or three-field lymph-node dissection, resulting in the sampling of a large number of lymph nodes: $48 \pm 21$ (mean \pm standard deviation). The number of dissected lymph nodes was not reported in most of the previous reports ${ }^{1-5}$ except for one, in which the mean number of examined lymph nodes was $14.1 .^{6}$ Thus, a precise comparison with previous reports is not feasible, as the number of examined lymph nodes differed. On the other hand, it is unlikely that patients preoperatively diagnosed as having LNM would be more likely to participate in the present analysis, as an esophagectomy with twoor three-field lymph-node dissection was performed regardless of the LNM diagnosis if surgery was selected for the treatment of SSCCE at our institution. ${ }^{13,14}$

In 2000 , we previously reported the results of uniand multivariate analyses to identify histopathological factors associated with LNM in 186 patients with M3 or SM lesions who underwent an esophagectomy and a two- or three-field lymph-node dissection. Only LY was independently associated with LNM in the multivariate analysis, and LY and HG were found to be significant prognostic factors in patients with SSCCE. None of the 21 patients with HG $1 / 2$ and a VTID from the muscularis mucosa of $<200 \mu \mathrm{m}$ without LY or V had LNM. LY, VTID and HG, therefore, were reported to be important factors for identifying patients who did not require addi- tional systemic treatment after EMR. In the present study, in which almost twice the number of cases was examined, LY was confirmed to be independently associated with LNM in patients with M3 and SM1 lesions. In addition, the depth of invasion (M3 vs SM1) turned out to be a significant factor for predicting LNM. We also classified the cases with M3 and SM1 lesions based on VTID, according to the method used in our previous report, but VTID was not significantly associated with LNM in this study.

In conclusion, we propose that SM lesions should be treated as a systemic disease. Only patients with M1/2 lesions are good candidates for EMR. M3 lesions with LY should be treated in the same way as SM lesions because M3 lesions with LY were associated with LNM.

\section{Acknowledgement}

We thank Dr Sandy Dawsey, National Institute of Health, Bethesda, MD, for his helpful comments on this manuscript.

\section{Duality of interest}

We have no duality of interest to declare.

\section{References}

1 Kodama M, Kakegawa T. Treatment of superficial cancer of the esophagus: a summary of responses to a questionnaire on superficial cancer of the esophagus in Japan. Surgery 1998;123:432-439.

2 Nabeya K, Nakata Y. Extent of resection and lymphadenectomy in early squamous cell esophageal cancer. Dis Esophagus 1997;10:159-161.

3 Tajima Y, Nakanishi Y, Ochiai A, et al. Histopathologic findings predicting lymph node metastasis and prognosis of patients with superficial esophageal carcinoma: analysis of 240 surgically resected tumors. Cancer 2000;88:1285-1293.

4 Fujita H, Sueyoshi S, Yamana H, et al. Optimum treatment strategy for superficial esophageal cancer: endoscopic mucosal resection vs radical esophagectomy. World J Surg 2001;25:424-431.

5 Shiozaki H, Doki Y, Yamana $\mathrm{H}$, et al. A multiinstitutional study of immunohistochemical investigation for the roles of cyclin D1 and E-cadherin in superficial squamous cell carcinoma of the esophagus. J Surg Oncol 2002;79:166-173.

6 Bogomoletz WV, Molas G, Gayet B, et al. Superficial squamous cell carcinoma of the esophagus. A report of 76 cases and review of the literature. Am J Surg Pathol 1989;13:535-546.

7 Sugimachi K, Ohno S, Matsuda H, et al. Clinicopathologic study of early stage esophageal carcinoma. Br J Surg 1989;76:759-763.

8 Bonavina L. Early oesophageal cancer: results of a European multicentre survey. Group Europeen pour 
l'Etude des Maladies de l'Oesophage. Br J Surg 1995;82:98-101.

9 Altorki NK, Skinner DB. Occult cervical nodal metastasis in esophageal cancer: preliminary results of three-field lymphadenectomy. J Thorac Cardiovasc Surg 1997;113:540-544.

10 Kato H, Tachimori Y, Watanabe H, et al. Superficial esophageal carcinoma. Surgical treatment and the results. Cancer 1990;66:2319-2323.

11 Matsubara T, Ueda M, Abe T, et al. Unique distribution patterns of metastatic lymph nodes in patients with superficial carcinoma of the thoracic oesophagus. Br J Surg 1999;86:669-673.
12 Travis WD, Brambilla E, Konrad Muller-Hermelink H, et al. World Health Organization Classification of Tumours. IARC Press: Lyon, 2000.

13 Igaki $\mathrm{H}$, Kato $\mathrm{H}$, Tachimori $\mathrm{Y}$, et al. Clinicopathologic characteristics and survival of patients with clinical Stage I squamous cell carcinomas of the thoracic esophagus treated with three-field lymph node dissection. Eur J Cardiothorac Surg 2001;20: 1089-1094.

14 Kato H, Tachimori Y, Mizobuchi S, et al. Cervical, mediastinal, and abdominal lymph node dissection (three-field dissection) for superficial carcinoma of the thoracic esophagus. Cancer 1993;72:2879-2882. 